\title{
Resonance region measurements of dysprosium and rhenium
}

\author{
Gregory Leinweber ${ }^{1, a}$, Robert C. Block ${ }^{1}$, Brian E. Epping ${ }^{1,5}$, Devin P. Barry ${ }^{1}$, Michael J. Rapp ${ }^{1}$, Yaron Danon ${ }^{2}$, \\ Timothy J. Donovan $^{1}$, Sheldon Landsberger ${ }^{5}$, John A. Burke ${ }^{1}$, Mary C. Bishop ${ }^{1,5}$, Amanda Youmans ${ }^{2}$, Guinyun N. Kim ${ }^{3}$, \\ yeong-rok Kang ${ }^{4}$, Man Woo Lee ${ }^{4}$, and Noel J. Drindak ${ }^{1-\text { ret. }}$ \\ 1 Bechtel Marine Propulsion Corp., Knolls Atomic Power Laboratory, PO Box 1072, Schenectady, NY 12301-1072, USA \\ 2 Rensselaer Polytechnic Institute, Gaerttner LINAC Center, $1108^{\text {th }}$ St., Troy, NY 12180, USA \\ 3 Department of Physics, Kyungpook National University, Daegu 702-701, Republic of Korea \\ ${ }^{4}$ Research Center, Dongnam Inst. Of Radiological \& Medical Sciences, Busan 619-953, Republic of Korea \\ 5 The University of Texas at Austin, Nuclear Engineering Teaching Lab, Pickle Research Campus, R-9000, Austin, Texas 78712, USA
}

\begin{abstract}
Neutron capture and transmission measurements have been performed, and resonance parameter analysis has been completed for dysprosium, Dy, and rhenium, Re. The $60 \mathrm{MeV}$ electron accelerator at RPI Gaerttner LINAC Center produced neutrons in the thermal and epithermal energy regions for these measurements. Transmission measurements were made using ${ }^{6} \mathrm{Li}$ glass scintillation detectors. The neutron capture measurements were made with a 16-segment $\mathrm{NaI}$ multiplicity detector. The detectors for all experiments were located at $\approx 25 \mathrm{~m}$ except for thermal transmission, which was done at $\approx 15 \mathrm{~m}$. The dysprosium samples included one highly enriched ${ }^{164}$ Dy metal, 6 liquid solutions of enriched ${ }^{164}$ Dy, two natural Dy metals. The Re samples were natural metals. Their capture yield normalizations were corrected for their high gamma attenuation. The multi-level R-matrix Bayesian computer code SAMMY was used to extract the resonance parameters from the data. ${ }^{164} \mathrm{Dy}$ resonance data were analyzed up to $550 \mathrm{eV}$, other Dy isotopes up to $17 \mathrm{eV}$, and Re resonance data up to $1 \mathrm{keV}$. Uncertainties due to resolution function, flight path, burst width, sample thickness, normalization, background, and zero time were estimated and propagated using SAMMY. An additional check of sample-to-sample consistency is presented as an estimate of uncertainty. The thermal total cross sections and neutron capture resonance integrals of ${ }^{164} \mathrm{Dy}$ and Re were determined from the resonance parameters. The NJOY and INTER codes were used to process and integrate the cross sections. Plots of the data, fits, and calculations using ENDF/B-VII.1 resonance parameters are presented.
\end{abstract}

\section{Introduction}

Natural dysprosium has a high neutron thermal capture cross section of 950 barns, approximately $80 \%$ of which is from capture in ${ }^{164} \mathrm{Dy} .{ }^{164} \mathrm{Dy}$ is produced inside a reactor as a fission product. It also can be used as a burnable poison to control a reactor [1]. Therefore, it is important to know the ${ }^{164}$ Dy cross section accurately.

Rhenium is a very rare material that does not exist in nature as a free element. It is extremely dense, has a high melting point, and has the highest boiling point of any element. Natural rhenium consists of ${ }^{185} \mathrm{Re}$ with a $37.40 \%$ abundance and ${ }^{187} \mathrm{Re}$, a long-lived isotope with half-life of $4.12 \times 10^{10}$ years and a $62.60 \%$ abundance. Rhenium is a refractory metal; i.e., extremely resistant to heat and wear; therefore, it has potential uses in reactor applications.

The Gaerttner LINAC Center at the Rensselaer Polytechnic Institute in Troy, New York has been used to perform neutron transmission and capture experiments to determine neutron resonance parameters and cross sections.

\section{Experimental setup}

Four experiments for each material were performed, transmission and capture in the thermal and epithermal

a e-mail: leinwg@rpi.edu energy ranges. Experimental parameters, targets, overlap filters, pulse widths, electron current, channel widths, burst rate, and flight paths were optimized for each experiment. A boron overlap filter was used for $\mathrm{Re}$ epithermal transmission and ${ }^{164} \mathrm{Dy}$ capture measurements. A cadmium overlap filter was used for Re epithermal capture and Dy epithermal transmission and capture. $\operatorname{Re}$ and Dy fluxes were normalized at the 2.16 and $5.44 \mathrm{eV}$ resonances, respectively, in the neutron capture experiments.

The detectors used in the transmission experiments were ${ }^{6} \mathrm{Li}$ glass viewed by photomultiplier tubes. The capture detector was a large 16-segment annulus of $\mathrm{NaI}$ that counted gamma rays emitted from neutron capture.[2] The detector surrounded the capturing sample and had $>85 \%$ efficiency to detect a capture event. The flight path length was $\approx 25 \mathrm{~m}$ for all experiments except thermal transmission at $15 \mathrm{~m}$ where energy resolution was less important.

The samples used in the Re measurements were disks of natural Re. The material is chemically benign and was measured as bare metal. The Dy metal samples were mildly reactive and were measured as bare metals. In addition, Dy liquid samples were prepared from dysprosium oxide enriched in ${ }^{164} \mathrm{Dy}$, dissolved in deuterated nitric acid, and housed in quartz cells. Several

(c) The Authors, published by EDP Sciences. This is an Open Access article distributed under the terms of the Creative Commons Attribution License 4.0 (http://creativecommons.org/licenses/by/4.0/). 

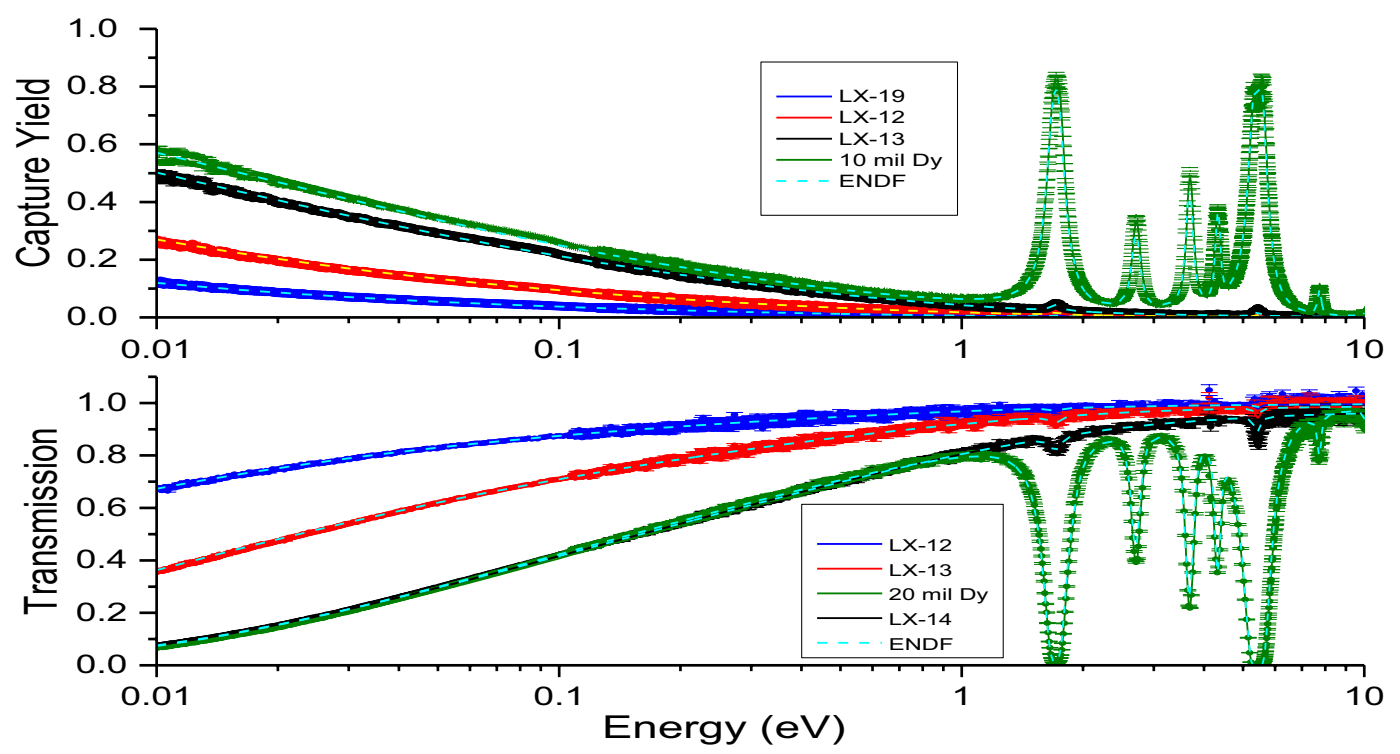

Figure 1. SAMMY fits to the thermal Dy data up to $10 \mathrm{eV}$. The resulting ${ }^{164} \mathrm{Dy}$ thermal cross section remains unchanged relative to ENDF, see Table 1 . The measured ${ }^{164}$ Dy capture resonance integral is slightly smaller than that given in ENDF/B-VII.1, see Table 2.

dilutions of the enriched Dy solution in heavy water provided the multiple sample thicknesses needed to get optimum transmission and capture yield and provide redundancy used to estimate uncertainties in the resulting resonance parameters.

\section{Data reduction}

The purpose of the data processing was to reduce the data to transmission, unitless fractions of samplein divided by sample-out counting rates, and neutron capture yields, expressing the fraction of incident neutrons which underwent neutron capture. All data were dead time corrected and compared to independent beam monitor detectors. The method for the reduction of the transmission and capture data was essentially the same as carried out by Leinweber et al. [2]

The neutron energy for each measured interaction is determined using the time-of-flight method. The zero time of the measurement is seen in the data as a burst of gamma rays coinciding with the burst of incident neutrons. A time spectrum of resulting interactions from each burst is recorded with a channel width of $0.03125 \mu \mathrm{s}$ for epithermal resonances, and up to $32 \mu \mathrm{s}$ at thermal energies where only broad structure exists.

The transmission background was normalized to a fixed notch in tungsten, at $\approx 18 \mathrm{eV}$, for the thermal measurements or manganese, at $\approx 330 \mathrm{eV}$, for the epithermal experiments. A fixed notch is a material with saturated resonance at the energy where the background function will be normalized. The fixed notch was present for all measurements. The background-subtracted samplein and "open beam" data were divided to generate the transmission data for each sample.

The critical step in the capture data processing was the normalization of the neutron flux. The neutron flux was determined by measuring the counting rate resulting from the placement of a thick $(2.54 \mathrm{~mm})$ enriched ${ }^{10} \mathrm{~B}$ carbide sample into the capture detector. The $478 \mathrm{keV}$ gamma emitted from the $(\mathrm{n} ; \alpha, \gamma)$ reaction was detected. The black resonances at $2.16 \mathrm{eV}$ in ${ }^{185} \mathrm{Re}$ and $5.44 \mathrm{eV}$ in ${ }^{162}$ Dy provided a region where the count rate is equal to the detector efficiency times the incident flux, since all incident neutrons react in a black resonance. Correction was made for fraction of interactions in each resonance which were scattered rather than captured. The capture counting rates were divided by the normalized neutron flux spectrum to generate capture yield data for each sample. Due to the high gamma attenuation of rhenium, its neutron flux normalization was sample thickness dependent. Capture detector relative efficiencies were determined for the Dy isotopes using results from Ref. [3].

\section{Results}

The SAMMY version 8 code [4] was used to extract resonance parameters from the transmission and capture yield data. SAMMY is a multi-level, R-matrix, Bayesian computer program that generates a single set of resonance parameters from multiple covariance matrix linked sample datasets. The code incorporates the effects of experimental resolution, Doppler broadening, self-shielding, and multiple-scattering. The isotopic assignments for all resonances were taken from ENDF/B-VII.1 [5].

The results of the SAMMY fits to the thermal Dy data are shown in Fig. 1. None of the low energy resonances shown are due to ${ }^{164} \mathrm{Dy}$. The lowest positive energy resonance in ${ }^{164} \mathrm{Dy}$ is at $147 \mathrm{eV}$. The results for that resonance are shown in Fig. 2. The set of resonance parameters shown is the result of fits to multiple samples in transmission and capture. The neutron width of the $147 \mathrm{eV}$ resonance is within the measurement uncertainty of the ENDF/B-VII.1 value. The radiation width is reduced significantly from the ENDF/B-VII.1 value. This is visible in the capture data fits in Fig. 2, which lie significantly below the ENDF curves. The measured thermal cross section of ${ }^{164}$ Dy is given in Table 1 and agrees with ENDF/B-VII.1 [5]. The thermal cross sections of ${ }^{164} \mathrm{Dy}$ and Re were processed from the fitted resonance parameters using NJOY [6] and integrated using the INTER [7] program. 


\begin{tabular}{|c|c|c|c|c|c|c|c|c|c|c|c|}
\hline Source & $\begin{array}{l}E_{0} \\
(e V)\end{array}$ & $\begin{array}{l}\Delta \mathrm{E}_{\mathrm{o}, \mathrm{B}} \\
(\mathrm{eV})\end{array}$ & $\begin{array}{l}\Delta E_{0, \text { ext }} \\
(e V)\end{array}$ & $\begin{array}{l}\Gamma_{v} \\
(\mathrm{meV})\end{array}$ & $\begin{array}{l}\Delta \Gamma_{V, 8} \\
(\mathrm{meV})\end{array}$ & $\begin{array}{l}\Delta \Gamma_{v, \text { ext }} \\
(\mathrm{meV})\end{array}$ & $\begin{array}{l}\Gamma_{n} \\
(\mathrm{meV})\end{array}$ & $\begin{array}{l}\Delta \Gamma_{n, 8} \\
(\mathrm{meV})\end{array}$ & $\begin{array}{l}\Delta \Gamma_{n, \text { ext }} \\
(\mathrm{meV})\end{array}$ & $\begin{array}{l}\text { Iso- } \\
\text { tope }\end{array}$ & J \\
\hline $\mathrm{BMPC} / \mathrm{RPI}$ & 147.105 & 0.005 & 0.008 & 86 & 146.97 & 1 & 830 & 8 & 10 & 164 & 0.5 \\
\hline ENDF/B-VII.1 & 147.97 & & & 114.2 & & & 820 & & & 164 & 0.5 \\
\hline
\end{tabular}
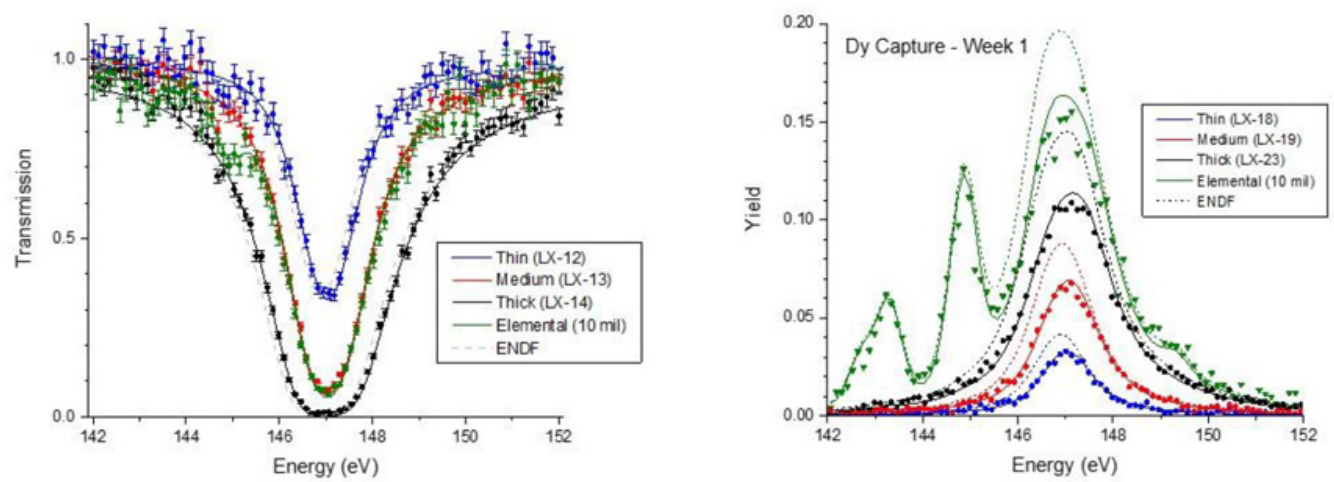

Figure 2. The $147 \mathrm{eV}$ resonance in ${ }^{164} \mathrm{Dy}$. This is the lowest positive energy resonance and the strongest resonance in ${ }^{164} \mathrm{Dy}$. The measured neutron width lies within its uncertainty of the ENDF value. Two types of uncertainties are given for the measured resonance parameters, Bayesian, $\Delta \mathrm{E}_{0, \mathrm{~B}}, \Gamma_{\gamma, \mathrm{B}}, \Delta \Gamma_{\mathrm{n}, \mathrm{B}}$, and external, based on consistency among samples, $\Delta \mathrm{E}_{0, \text { ext }}, \Gamma_{\gamma, \text { ext }}, \Delta \Gamma_{\mathrm{n} \text {,ext }}$. The radiation width is lower than ENDF, as seen in the capture fits to the first week of capture data. The parameters were derived from the data shown in addition to the $2^{\text {nd }}$ week of capture data (not shown).

Table 1. The measured thermal total cross section for ${ }^{164} \mathrm{Dy}$.

\begin{tabular}{|c|c|}
\hline \multicolumn{2}{|c|}{${ }^{164}$ Dy Thermal Total Cross Section } \\
\hline BMPC/RPI & ENDF \\
\hline $2980 \pm 10$ barns & 2981 barns \\
\hline
\end{tabular}

Table 2. The measured capture resonance integral for ${ }^{164} \mathrm{Dy}$.

\begin{tabular}{|c|c|}
\hline \multicolumn{2}{|c|}{${ }^{164}$ Dy Capture Resonance Integral } \\
\hline BMPC/RPI & ENDF \\
\hline $338 \pm 1$ barns & 342.2 barns \\
\hline
\end{tabular}

The capture resonance integral is defined in Eq. (1),

$$
R I_{\gamma}=\int_{0.5 e V}^{20 M e V} \sigma_{\gamma}(E) \frac{d E}{E} .
$$

The measured capture resonance integral of ${ }^{164} \mathrm{Dy}$ is given in Table 2 and is slightly smaller than that of ENDF/B-VII.1. The BMPC/RPI values were calculated from the measured resonance parameters using the NJOY and INTER programs. ${ }^{164}$ Dy resonances were fitted up to $540 \mathrm{eV}$. ENDF/B-VII.1 resonance parameters were used for all resonances above the measured energy range.

The uncertainties in the thermal total cross sections and capture resonance integrals were determined using a Monte Carlo method by sampling from the maximum uncertainties, either Bayesian or external (see Sect. 4.1), whichever is larger, of the resonance parameters and repeating the NJOY and INTER runs using the sampled parameters until sufficient statistics were obtained.

Fits to the thermal region in Re are shown in Fig. 3. The lowest positive energy resonance occurs at $2.1566 \mathrm{eV}$. Its resonance parameters are given in the figure. Both the neutron and radiation widths are somewhat reduced compared to the ENDF/B-VII.1 values. The uncertainties in the resonance energy and the neutron width are the larger of the Bayesian or external uncertainties. The radiation width and its uncertainty were fitted from all data from ${ }^{185} \mathrm{Re} \mathrm{J}=3$ resonances that contained radiation width information.

The thermal capture and total cross sections for rhenium are given in Table 3 . The methods for the $\mathrm{Re}$ analysis were the same as those for Dy. The measured thermal cross sections are nearly unchanged from the ENDF/B-VII.1 values. Rhenium resonances were fitted up to $1 \mathrm{keV}$. The rhenium capture resonance integrals are given in Table 4 . The measured resonance integrals of both isotopes are larger than those given by ENDF/B-VII.1.

Neutron strength functions, $\mathrm{S}_{0}$, for rhenium are given in Table 5. The BMPC/RPI values were derived from measured neutron widths over the energy region up to $200 \mathrm{eV}$ for ${ }^{185} \mathrm{Re}$ and $270 \mathrm{eV}$ for ${ }^{187} \mathrm{Re}$, where the observed level density is approximately constant. The measured strength for ${ }^{187} \mathrm{Re}$ is approximately $1 \sigma$ larger than that given by ENDF/B-VII.1 and the Atlas of Neutron Resonances [8].

\subsection{Uncertainty analysis}

Uncertainties due to resolution function, flight path, burst width, sample thickness, normalization, background, and zero time were estimated and propagated using SAMMY. Some components of uncertainty were underestimated. The Bayesian treatment of input uncertainties of resonance parameters masks the effect of the uncertainty in capture flux normalization. The treatment of background in transmission as an additive constant to transmission underestimates its effect on resonance widths. And the inability to propagate inconsistencies between individual sample spectra leaves a large contributor to the overall uncertainty unquantified. 

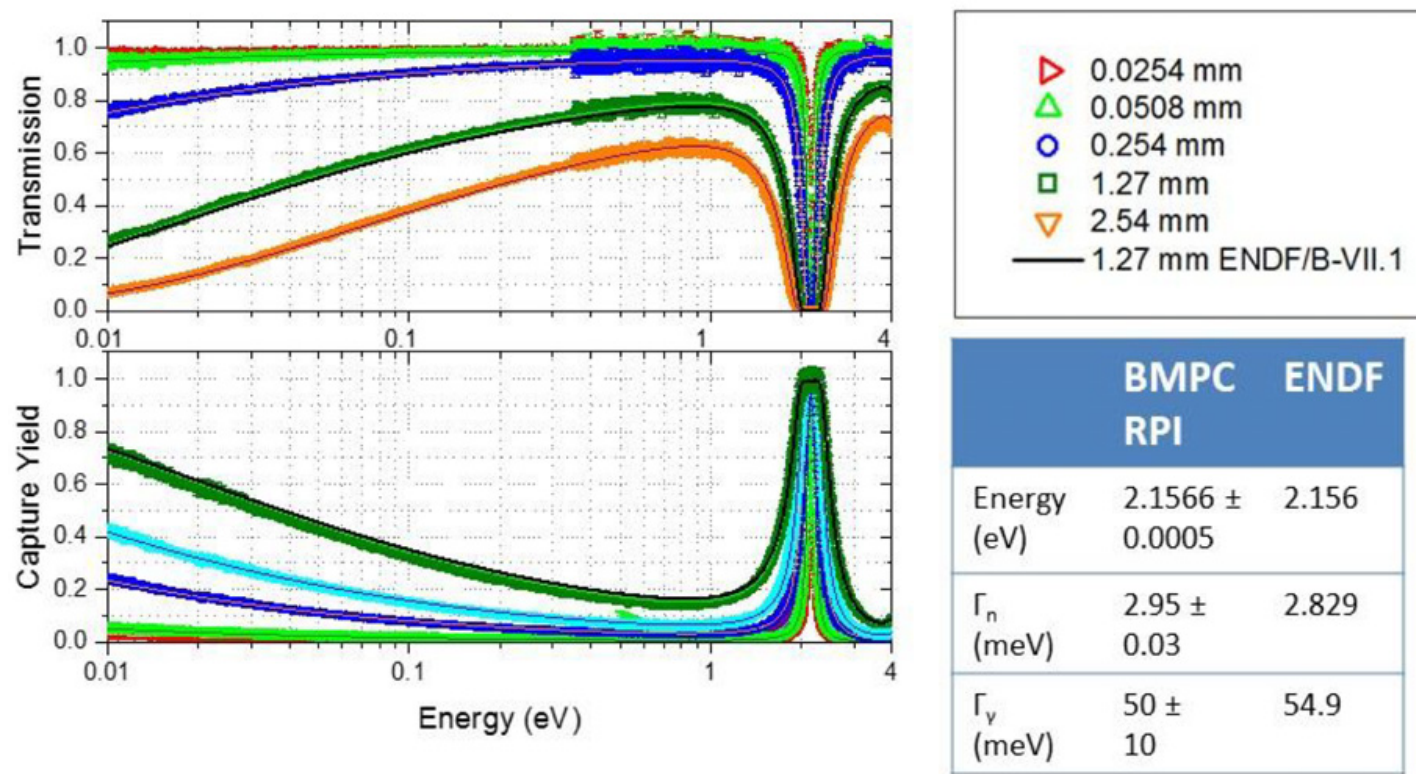

Figure 3. Thermal fits to Re data including the $2.16 \mathrm{eV}$ resonance. The $2.16 \mathrm{eV}$ resonance parameters in the bottom right table were derived from both thermal and epithermal (not shown) data. The uncertainties are described in the text.

Table 3. Thermal cross sections for the rhenium isotopes.

\begin{tabular}{|l|c|c|c|c|}
\hline & \multicolumn{2}{|c|}{${ }^{185} \mathrm{Re}$} & \multicolumn{2}{c|}{${ }^{187}$ Re } \\
\hline & $\begin{array}{c}\text { Thermal } \\
\text { Capture } \\
\text { Cross } \\
\text { Section } \\
\text { (barns) }\end{array}$ & $\begin{array}{c}\text { Thermal } \\
\text { Total } \\
\text { Cross } \\
\text { Section } \\
\text { (barns) }\end{array}$ & $\begin{array}{c}\text { Thermal } \\
\text { Capture } \\
\text { Cross } \\
\text { Section } \\
\text { (barns) }\end{array}$ & $\begin{array}{c}\text { Thermal } \\
\text { Total } \\
\text { Cross } \\
\text { Section } \\
\text { (barns) }\end{array}$ \\
\hline $\begin{array}{l}\text { BMPC/ } \\
\text { RPI }\end{array}$ & $110 \pm 2$ & $118 \pm 2$ & $75 \pm 3$ & $82 \pm 4$ \\
\hline $\begin{array}{l}\text { Arboccò } \\
\text { et al. [9] }\end{array}$ & $111.6 \pm 1.1$ & N/A & $74.6 \pm 1.4$ & N/A \\
\hline $\begin{array}{l}\text { ENDF/ } \\
\text { B-VII.1 }\end{array}$ & 112 & 121 & 77 & 87 \\
\hline $\begin{array}{l}\text { BMPC/ } \\
\text { RPI Percent } \\
\text { change } \\
\text { from } \\
\text { ENDF/ } \\
\text { B-VII.1 }\end{array}$ & $-2 \pm 2$ & $-2 \pm 2$ & $-3 \pm 4$ & $-6 \pm 5$ \\
\hline
\end{tabular}

Table 4. Capture resonance integrals for the rhenium isotopes cross sections for the rhenium isotopes.

\begin{tabular}{|l|c|c|}
\hline & $\begin{array}{c}{ }^{185} \text { Re Capture } \\
\text { Resonance Integral } \\
\text { (barns) }\end{array}$ & $\begin{array}{c}{ }^{187} \text { Re Capture } \\
\text { Resonance Integral } \\
\text { (barns) }\end{array}$ \\
\hline BMPC/RPI & $1800 \pm 20$ & $310 \pm 3$ \\
\hline ENDF/B-VII.1 & 1738 & 301 \\
\hline $\begin{array}{l}\text { Percent change } \\
\text { from ENDF/ } \\
\text { B-VII.1 }\end{array}$ & $+4 \pm 1$ & $+3 \pm 1$ \\
\hline
\end{tabular}

Therefore, an additional check of sample-to-sample consistency was performed as an estimate of uncertainty. Although the experimental parameters responsible for these variations were not determined their overall impact was quantified. A separate SAMMY fit using the best estimates of experimental uncertainty was performed for each individual sample in each experimental configuration. A weighted standard deviation of the distribution of
Table 5. Neutron strength function, $S_{0}$, for the naturallyoccurring isotopes of Re. The uncertainties for ENDF are estimated using the relative uncertainties from the Atlas of Neutron Resonances [8] since no uncertainties were available for the neutron widths.

\begin{tabular}{|l|l|l|}
\hline & $\begin{array}{l}\mathrm{S}_{0}^{185} \mathrm{Re} \\
\left(10^{-4} \mathrm{x} \mathrm{meV}^{-1 / 2}\right)\end{array}$ & $\begin{array}{l}\mathrm{S}_{0}^{187} \mathrm{Re} \\
\left(10^{-4} \mathrm{x} \mathrm{meV}^{-1 / 2}\right)\end{array}$ \\
\hline BMPC/RPI & $1.5 \pm 0.1$ & $3.1 \pm 0.1$ \\
\hline ENDF/B-VII.1 & $1.5 \pm 0.3$ & $2.6 \pm 0.4$ \\
\hline $\begin{array}{l}\text { Atlas of } \\
\text { Neutron } \\
\text { Resonances [8] }\end{array}$ & $2.16 \pm 0.14$ & $2.43 \pm 0.28$ \\
\hline
\end{tabular}

outcomes from the individual sample fits was quoted as the "external" uncertainty. The larger of the Bayesian or external uncertainty for each parameter is the one quoted through this document.

\section{Conclusions}

The RPI electron accelerator was used to produce neutrons used in transmission and capture experiments. Measurements in the thermal and epithermal range were made on samples of Dy and Re. Liquid samples of ${ }^{164}$ Dy were fabricated to get uniform, thin samples suitable for measuring a large thermal cross section without saturation. A combination of standard uncertainty propagation, Monte Carlo sampling within uncertainties, and weighted standard deviations of distributions of outcomes from individual sample fits was used to determine the uncertainties on the reported parameters. Final results include the resonance parameters of the lowest positive energy resonance of ${ }^{164}$ Dy and ${ }^{185} \mathrm{Re}$, thermal cross sections, and capture resonance integrals. The thermal total cross section of ${ }^{164} \mathrm{Dy}$ is unchanged from the ENDF/B-VII.1 value. The thermal total cross sections of both of the rhenium isotopes are slightly lower than those of ENDF/B-VII.1. The capture resonance integral of ${ }^{164}$ Dy is slightly smaller than that of ENDF/B-VII.1. The 
capture resonance integrals of both of the rhenium isotopes are larger than those of ENDF/B-VII.1.

\section{References}

[1] W.A. Raaijmakers, European Atomic Energy Community report EUR 2184.e (1965)

[2] G. Leinweber, D.P. Barry, J.A. Burke, M.J. Rapp, R.C. Block, Y. Danon, J.A. Geuther, F.J. Saglime III, Ann. Nucl. Energy 69, 74 (2014)

[3] S. Wang, M. Lubert, Y. Danon, N.C. Francis, R.C. Block, F. Becvar, M. Kriticka, Nucl. Instrum Meth. A 513, 585 (2003)
[4] N.M. Larson, ORNL/TM-9179/R8 ENDF-364/R2, Oak Ridge National Laboratory (2008)

[5] M.B. Chadwick et al., Nuclear Data Sheets 112, 2887 (2011)

[6] R.E. MacFarlane, D.W. Muir, LA-12740-M, Los Alamos National Laboratory (1994)

[7] C.L. Dunford, "ENDF Utility Codes Release 6.12," Informal Report (2001)

[8] S.F. Mughabghab, Atlas of Neutron Resonances, 5th Edition, Elsevier, New York (2006)

[9] F. Farina Arbocco, P. Vermaercke, K. Smits, L. Sneyers, K. Strijckmans, J. Radioanal. Nucl. Ch. 296, 931 (2013) 